\title{
Crystal Deposition Disease and Psoriatic Arthritis
}

\author{
Philip J. O'Connor, MRCP, FRCR, FFSEM (UK) $)^{1,2}$ \\ ${ }^{1}$ Department of Radiology, Leeds Teaching Hospitals, Chapel Allerton \\ Hospital, Leeds, United Kingdom \\ 2 Leeds Musculoskeletal Biomedical Research Unit, Leeds Teaching \\ Hospitals, Chapel Allerton Hospital, Leeds, United Kingdom
}

\begin{abstract}
Address for correspondence Philip J. O'Connor, MRCP, FRCR, FFSEM (UK), Department of Radiology and Leeds Musculoskeletal Biomedical Research Unit, Leeds Teaching Hospitals, Chapel Allerton Hospital, Chapeltown Road, Leeds LS7 4SA, UK (e-mail: Philip.OConnor@leedsth.nhs.uk).
\end{abstract}

Semin Musculoskelet Radiol 2013;17:74-79.

\begin{abstract}
Keywords

- gout

- pseudogout

- CPPD

- psoriatic arthritis

To the practicing clinical radiologist, knowledge of the ultrasound appearances of crystal diseases is important. Assessment of bone or soft tissue changes related to rheumatoid arthritis is a rare clinical indication for ultrasound, whereas crystal diseases have a broad spectrum of presentations requiring clinical diagnostic imaging. Psoriatic arthritis is an entheseal disease with secondary joint involvement, where imaging findings include inflammatory enthesopathy with erosions as well as flexor tendon tenosynovitis. The ultrasound features of crystal deposition diseases and psoriatic arthritis in soft tissues and joints are relatively characteristic, and an awareness of these appearances is vital to the practicing musculoskeletal sonologist.
\end{abstract}

\section{Gout}

Gout is a potentially destructive inflammatory disorder occurring in the setting of hyperuricemia. Urate crystals are deposited into the soft tissues or joint where they can produce either an acute neutrophil-mediated inflammatory reaction or a more chronic macrophage-mediated response. The variation in response is thought to be the result of differing crystal sizes with larger crystals failing to cause acute inflammatory reactions. Smaller proinflammatory crystals tend to be seen when there are rapid changes in serum urate levels.

Hyperuricemia is elevated levels of serum urate, which is the end product of purine metabolism. The physiologic definition of hyperuricemia is levels $>6.8 \mathrm{mg} / \mathrm{dL}$ ( $402 \mu \mathrm{mol} /$ L); this is the saturation point of sodium urate salt in biological tissues. Asymptomatic hyperuricemia is common, occurring in $\sim 19 \%$ of individuals in the United States and United Kingdom. Studies show that this hyperuricemic state can persist for decades with the Framingham study showing only 1 in 8 of patients with urate levels between 7 and $8 \mathrm{mg} /$ $\mathrm{dL}$ developing clinical gout over a 14 -year period. ${ }^{1}$ Factors that increase the likelihood of developing clinical gout are blood urate level $>9 \mathrm{mg} / \mathrm{dL}$ and comorbidities such as renal failure, cardiovascular disease, obesity, and diabetes.

Gout is much more common in men than women with 95\% of all cases male. Men normally develop gout in their fourth to sixth decades with female gout occurring postmenopause. Certain ethnic groups have a predilection for developing gout with high incidences of the disease in Pacific Islanders and Filipino patients. Gout initially presents as acute intermittent gout progressing to chronic tophaceous gout that in itself manifests several differing clinical presentations.

\section{Acute Intermittent Gout}

Patients present with severe pain, erythema, and swelling in the affected joint, which is usually in the lower limb. In $~ 50 \%$ of cases, this is the first metatarsophalangeal joint, but the midfoot, ankle, and knee are also commonly involved. The presentation can frequently mimic infection. Frequently aspiration to exclude infection is required with microscopy to confirm or refute the presence of infection.

In imaging terms, patients have effusion and synovitis in the affected joint with fine hyperechoic foci seen either within the effusion, synovium, or on the cartilage surface

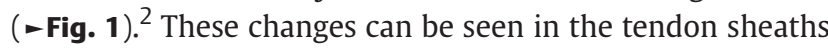
especially around the hand and wrist (-Fig. 2). Such hyperechoic foci can be seen in patients with asymptomatic hyperuricemia. These patients commonly lay down crystals in or around joints for a substantial period of time before finally presenting with gout. Crystals are seen at ultrasound in up to a third of these patients. ${ }^{3}$ As a result it is best to treat the identification of these foci as indicative but not diagnostic of acute gout.
Issue Theme Musculoskeletal Ultrasound Update; Guest Editor, Jon A. Jacobson, MD.
Copyright @ 2013 by Thieme Medical Publishers, Inc., 333 Seventh Avenue, New York, NY 10001, USA. Tel: +1(212) 584-4662.
DOI http://dx.doi.org/ 10.1055/s-0033-1333940. ISSN 1089-7860. 
a
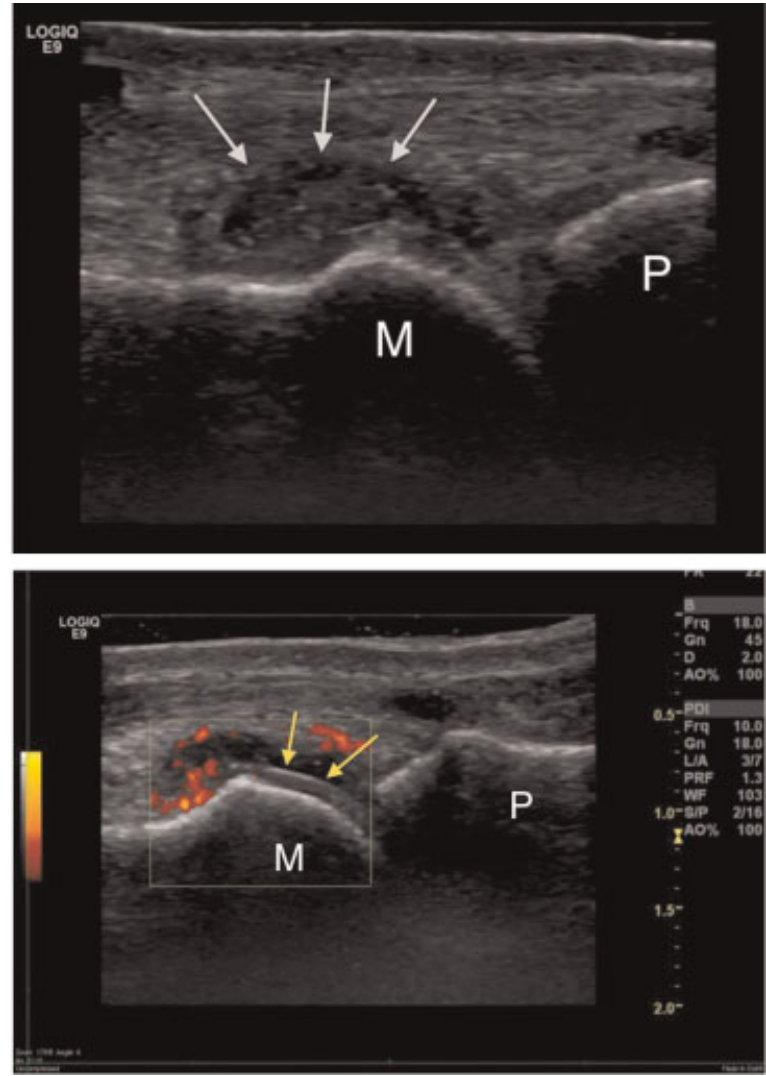

Fig. 1 Longitudinal sonogram of the first metatarsophalangeal joint of patient with acute gout. (a) Grayscale image shows effusion and synovitis containing hyperechoic foci (arrows). (b) Power Doppler image shows hypervascularity. A double contour sign is present on the cartilage surface as the result of urate crystal deposition (arrows). M, metatarsal head; P, phalangeal base.

One advantage of ultrasound is being able to take a clinical history during the examination. With regard to gout, it is often required to ask directly if the patient has gout. For some reason it is commonly overlooked by patients who do not volunteer the information when asked if they have any other medical conditions. Possible explanations include that gout may have some perceived stigma of a decadent lifestyle or is simply not seen as a medical condition by patients.

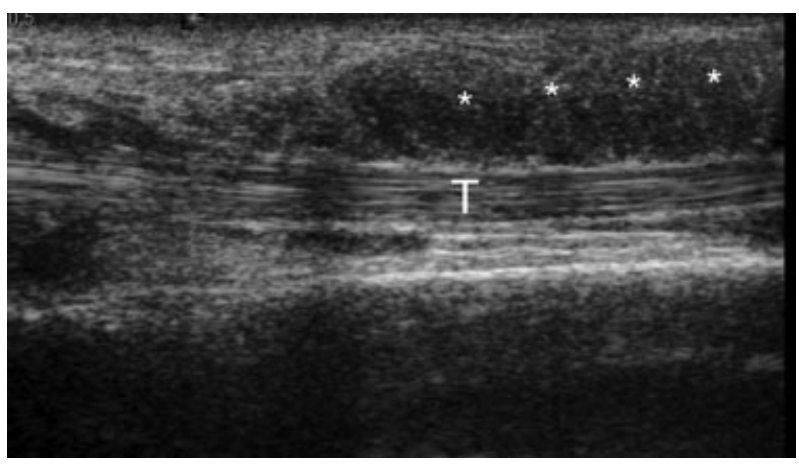

Fig. 2 Longitudinal grayscale ultrasound of the extensor tendons of the wrist $(\mathrm{T})$ shows extensive complex fluid containing hyperechoic foci (asterisks).

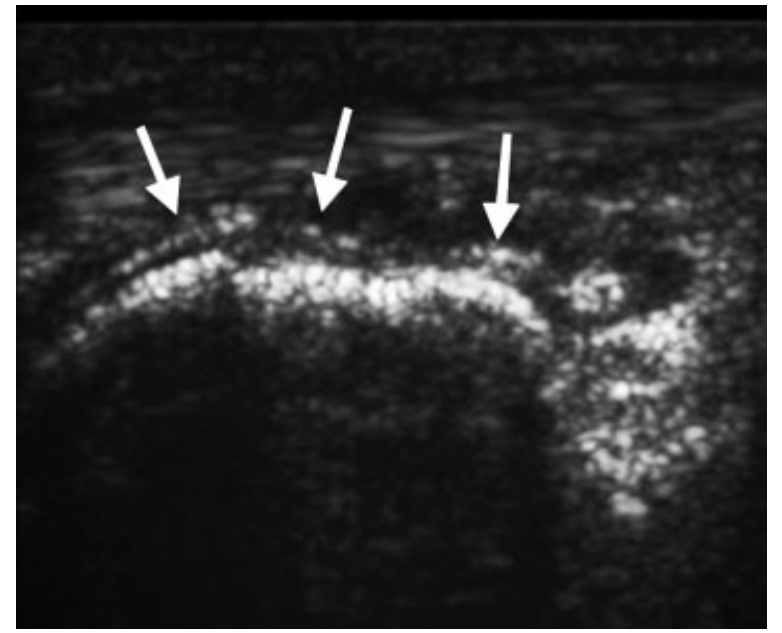

Fig. 3 Longitudinal grayscale ultrasound of the metacarpophalangeal joint in a patient with gout shows urate crystal deposition on the cartilage surface of the metatarsal head (arrows).

\section{Chronic Tophaceous Gout}

When affected joints are no longer pain free between attacks, the patient has developed chronic gouty arthritis. Chronic crystal deposition occurs in both the joints and soft tissues.

Joint changes involve effusion, synovitis, and erosion. Crystal deposition within synovium and on the cartilage surface is a prominent feature with a double contour sign described as a feature of cartilage surface crystal deposition (-Fig. 3). ${ }^{4}$ The erosions seen in chronic gout are characteristically periarticular with a broad base and overhanging margins (-Fig. 4). Studies have shown, however, that

a
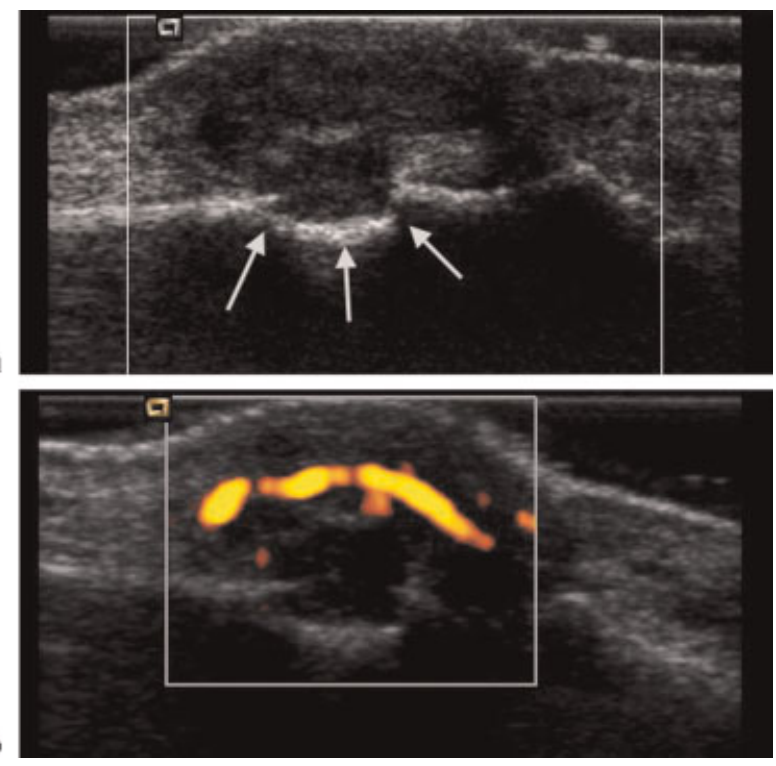

Fig. 4 Longitudinal grayscale sonogram of a patient with chronic tophaceous gout. (a) Ultrasound image shows typical appearances of an erosion with overhanging margins (arrows). (b) Power Doppler image in the same patient shows hypervascular periarticular inflammatory change. 
a
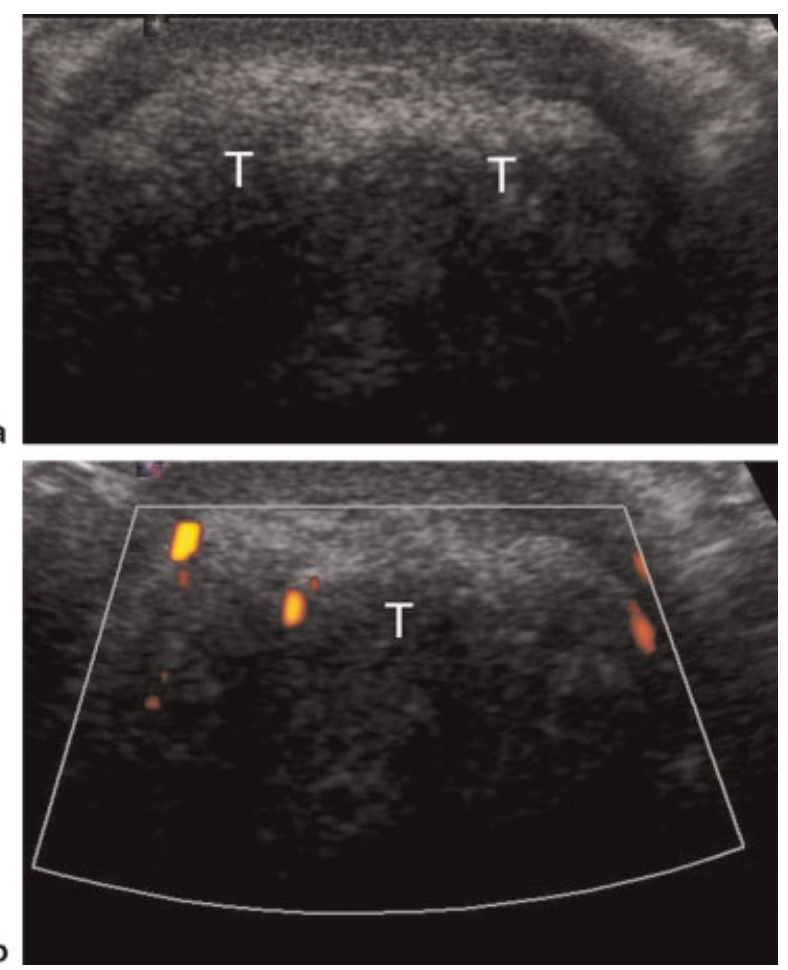

Fig. 5 (a) Grayscale and (b) power Doppler images show typical appearances of a tophus ( $T$ ) with hyperechoic heterogeneous echotexture, acoustic shadowing, and low-grade neovascularity.
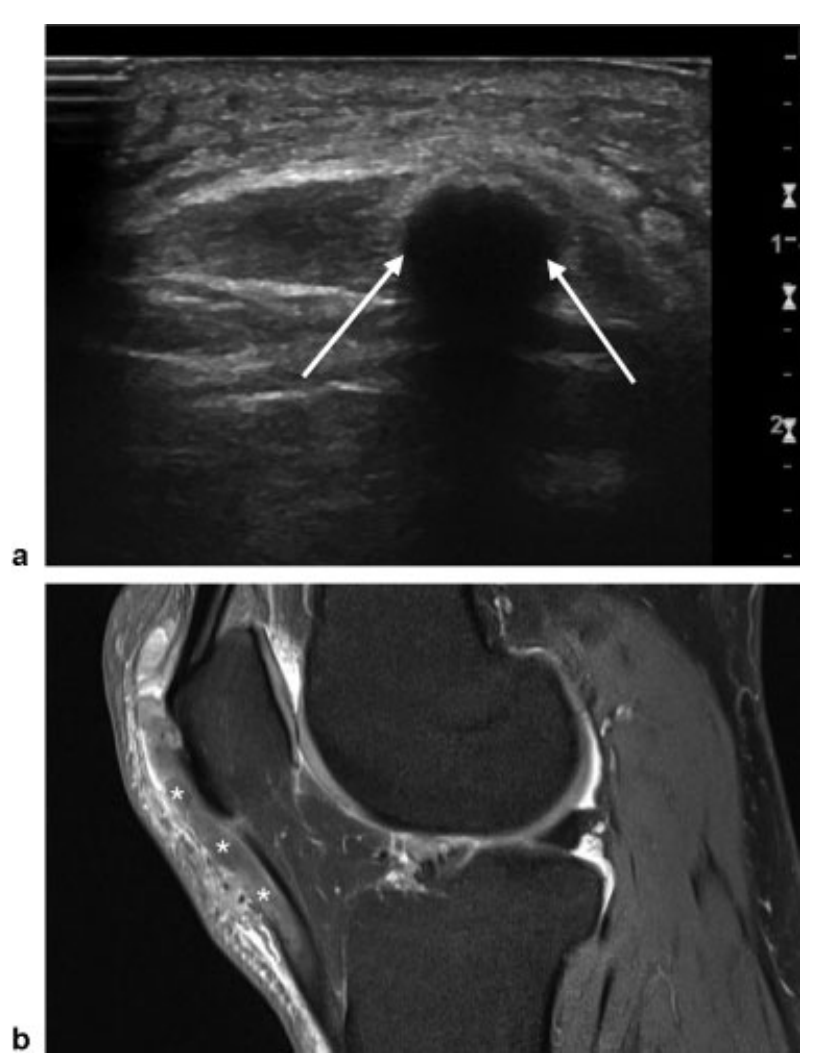

Fig. 6 (a) Transverse grayscale sonogram of the patellar tendon shows central urate crystal deposition (arrows). (b) Proton-density fat-saturated sagittal MR image in same patient shows urate crystal deposition that extends down the length of the tendon (asterisks). ultrasound is insensitive when compared with MR at demonstrating bone changes. ${ }^{5}$

Soft tissue crystal deposition is common in chronic gout. Tophi are the sentinel feature of chronic gout and are soft tissue masslike lesions occurring most commonly in the hands and wrist (ulna aspect) and on the extensor surface of the knees and elbows. Tophi can be clinically confused with other arthritis-related nodules such as rheumatoid nodules or other deposition arthritides such as multicentric reticulohistiocytosis. In contrast to tophi, rheumatoid nodules are typically hypoechoic.

Tophi have characteristic ultrasound imaging features with lesions lying in the subcutaneous compartment associated with well-organized monosodium urate crystal deposition within the mass. In imaging terms, this produces hazy somewhat ill-defined heterogeneous hyperechoic lesions associated with acoustic shadowing ( - Fig. 5). A hypoechoic halo is seen around $50 \%$ of tophi. ${ }^{6}$

Tendon crystal deposition is also common in tophaceous gout. Typically tendon involvement extends from a subcutaneous tophus with hyperechoic heterogeneous material that extends longitudinally along within the tendon substance (-Fig. 6).Tendon involvement can also be entheseal; demonstration of hyperechoic foci with the area of tendinopathy and erosion should alert the examiner to the possibility of goutrelated tendon disease. This entheseal tendon disease typically shows marked hypervascularity ( - Fig. $\mathbf{7}$ ).
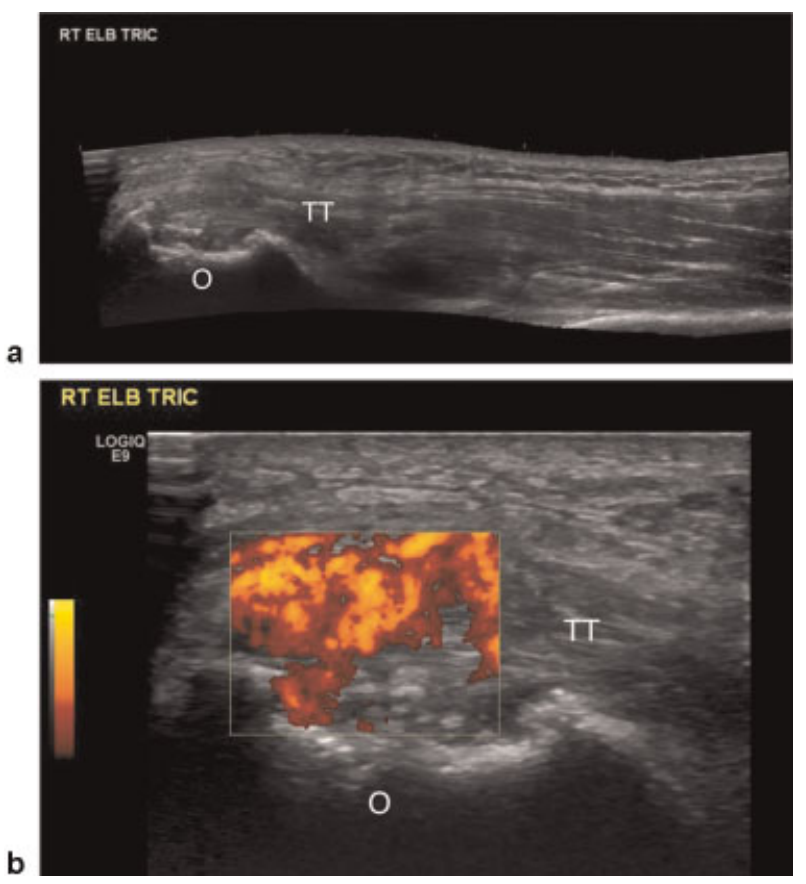

Fig. 7 (a) Longitudinal grayscale sonogram of the triceps tendon (TT) shows erosion of the olecranon $(\mathrm{O})$. The tendon contains numerous echogenic foci typical for gout-related entheseal involvement. (b) Power Doppler image of the same triceps tendon shows marked neovascularity. 


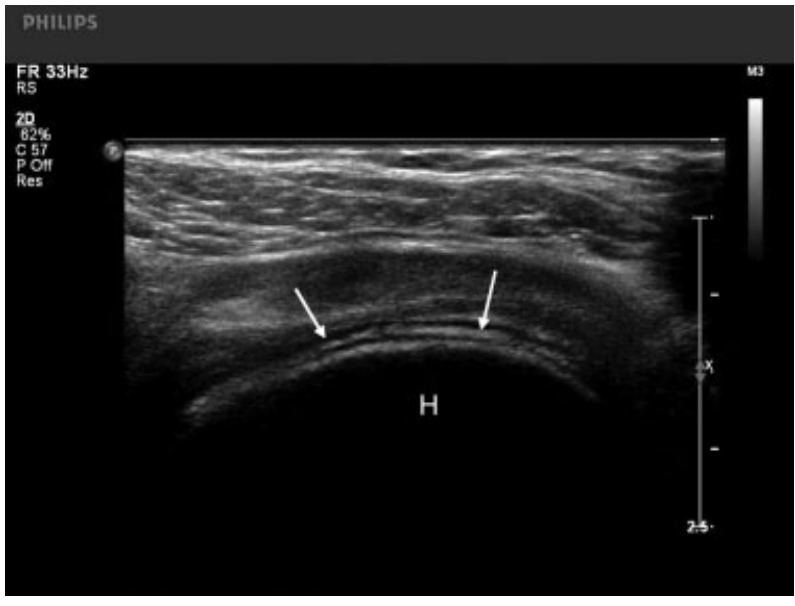

Fig. 8 Grayscale sonogram of the humeral head $(\mathrm{H})$ shows typical calcium pyrophosphate dihydrate deposition within the cartilage of the humeral head (arrows).

\section{Pseudogout}

Calcium pyrophosphate dihydrate deposition (CPPD) disease can result in crystal deposition within articular and periarticular tissues that is associated with cartilage calcification (chondrocalcinosis), joint inflammation (pseudogout), and joint destruction (CPPD arthropathy). ${ }^{7}$ Calcium pyrophosphate dihydrate crystals can also be seen on ultrasound examination. They have virtually identical features to urate crystals, although they have a slightly different distribution
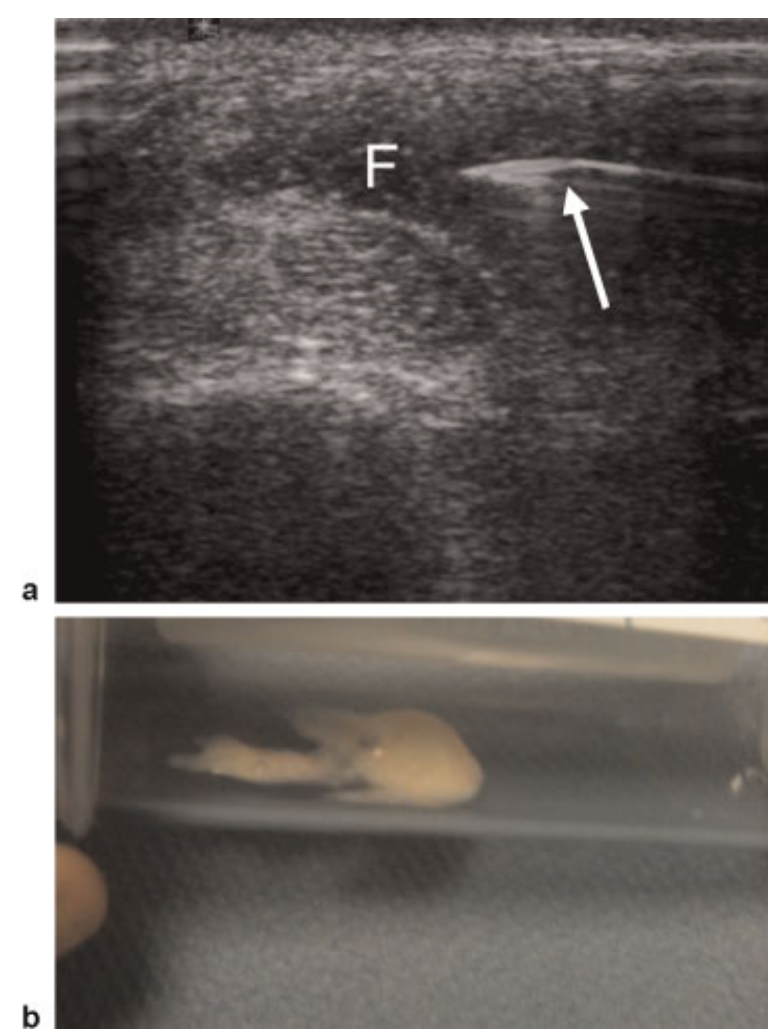

Fig. 9 (a) Image of needle placement (arrow) within fluid-containing echogenic foci (F). (b) Aspirate of fluid showing typical milky appearance seen in gout. being more within rather than on the surface of the articular cartilage (-Fig. 8). ${ }^{8}$ Unfortunately, patients with acute pseudogout get crystal shedding into the joint that may appear similar to the ultrasound appearance of acute gout. Thus aspiration and microscopy of joint fluid is often required in these cases to distinguish gout and pseudogout, especially when the patient has borderline hyperuricemia (-Fig. 9).

\section{Psoriatic Arthritis}

The link between psoriasis and arthritis is complex. Patients with inflammatory arthritis have an increased incidence of psoriasis, and patients with psoriasis have an increased incidence of inflammatory arthropathy as well. What complicates matters are reports of an increased incidence of rheumatoid arthritis in psoriatic patients. ${ }^{9}$ Once seropositive patients are removed, the link between psoriasis and inflammatory arthritis is stronger; $20 \%$ of patients with seronegative inflammatory arthritis have psoriasis. ${ }^{10}$

Psoriatic arthritis is thought to be an entheseal disease with secondary joint involvement. ${ }^{11}$ In the hands and feet it has an asymmetric distal distribution most commonly involving the distal interphalangeal joints.

The ultrasound features of foot and hand involvement include enthesitis at the flexor and extensor insertions (-Fig. 10) on the distal phalanx characterized by simultaneous erosion and new bone formation. This is an unusual combination in untreated rheumatoid arthritis in that the erosions are not associated with new bone formation until patients are in remission and begin developing secondary osteoarthritis.

The classical sausage shape digit of dactylitis occurs in $\sim 40 \%$ of patients with psoriatic arthritis. Although joint disease does contribute to the development of finger swelling, flexor tendon tenosynovitis is the most common pathology leading to dactylitis. ${ }^{12}$ This has a characteristic nodular/ irregular appearance on ultrasound with tenosynovial sheath fluid and solid hyperemic nodules present (-Fig. 11).

Large joint involvement from psoriatic arthritis tends to involve only one or two joints with diffuse low-grade synovitis and effusion most commonly seen in the lower limbs. This mono- or oligoarthritic presentation like this is more common in male patients and has very nonspecific ultrasound findings.

Symptomatic enthesitis is seen in up to $40 \%$ of patients with psoriatic arthritis and is a presenting feature in $4 \%$. The reported incidence of asymptomatic enthesitis seen in psoriatic arthritis patients varies from $4 \%{ }^{13}$ to as high as $50 \%{ }^{14}$ The tendon ultrasound features of enthesitis in psoriatic arthritis are nonspecific with tendinopathy seen close to the insertion or origin of tendon. Involved tendons have increased thickness, hypoechogenicity, and neovascularity, which can be associated with erosion of the adjacent bone (-Fig. 12). From a clinical viewpoint, entheseal tendon disease is rare in young skeletally mature patients, so it is important to assess carefully for any associated entheseal erosion or new bone formation in such patients who present with tendon disease close to the enthesis. 

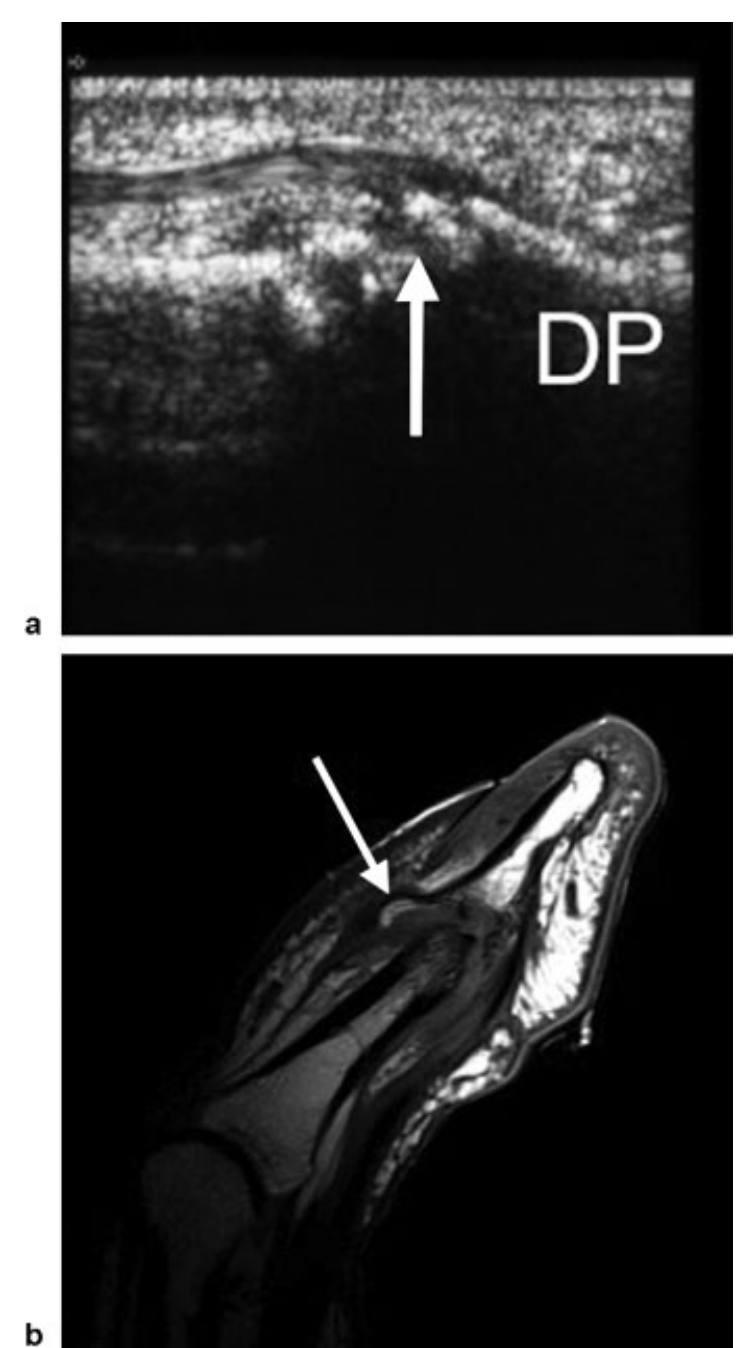

Fig. 10 (a) Longitudinal sonogram of the extensor tendon inserting on the distal phalanx (DP) in a patient with psoriatic arthritis shows simultaneous erosion and new bone formation (arrow). (b) T1weighted MR image of DP of a patient with psoriatic arthritis shows pencil-in-cup deformity and new bone formation at the extensor tendon insertion (arrow).

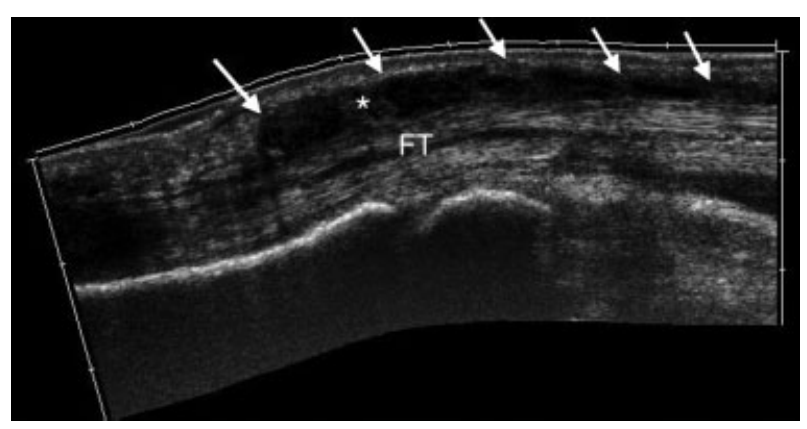

Fig. 11 Extended field-of-view longitudinal sonogram of a patient with psoriatic dactylitis. Extensive flexor tendon (FT) tenosynovitis (arrows) containing solid nodules (asterisk) is present.

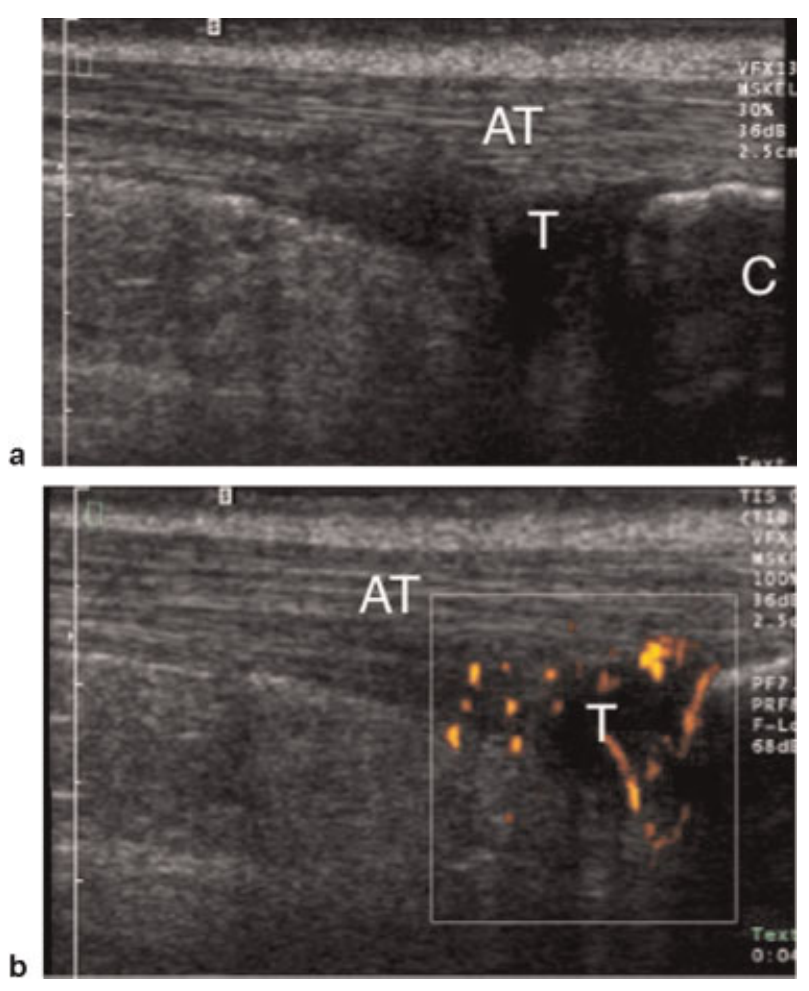

Fig. 12 (a) Grayscale and (b) power Doppler images of the distal Achilles tendon (AT) show typical psoriatic entheseal disease with distal Achilles tendinopathy $(T)$ with thickening, hypoechogenicity, and neovascularity of the tendon and adjacent retrocalcaneal bursitis.

\section{Key Points}

1. Crystal diseases can present in several ways from an acutely inflamed joint to a mass lesion.

2. Crystals can be seen with musculoskeletal ultrasound.

3. Urate crystals can be present in patients with asymptomatic hyperuricemia and as such should be viewed as indicative but not diagnostic of gout.

4. Psoriatic arthritis results most likely results from enthesitis with secondary intra-articular inflammation.

5. The primary imaging finding in psoriatic dactylitis is nodular flexor tenosynovitis.

\section{References}

1 Abbott RD, Brand FN, Kannel WB, Castelli WP. Gout and coronary heart disease: the Framingham Study. J Clin Epidemiol 1988;41 (3):237-242

2 Thiele RG. Role of ultrasound and other advanced imaging in the diagnosis and management of gout. Curr Rheumatol Rep 2011;13 (2):146-153

3 De Miguel E, Puig JG, Castillo C, Peiteado D, Torres RJ, Martín-Mola E. Diagnosis of gout in patients with asymptomatic hyperuricaemia: a pilot ultrasound study. Ann Rheum Dis 2012;71(1):157158

4 Filippucci E, Riveros MG, Georgescu D, Salaffi F, Grassi W. Hyaline cartilage involvement in patients with gout and calcium pyrophosphate deposition disease. An ultrasound study. Osteoarthritis Cartilage 2009;17(2):178-181

5 Carter JD, Kedar RP, Anderson SR, et al. An analysis of MRI and ultrasound imaging in patients with gout who have normal plain radiographs. Rheumatology (Oxford) 2009;48(11):14421446 
6 de Ávila Fernandes E, Kubota ES, Sandim GB, Mitraud SA, Ferrari AJ, Fernandes AR. Ultrasound features of tophi in chronic tophaceous gout. Skeletal Radiol 2011;40(3):309-315

7 Bencardino JT, Hassankhani A. Calcium pyrophosphate dihydrate crystal deposition disease. Semin Musculoskelet Radiol 2003;7 (3):175-185

8 Filippucci E, Riveros MG, Georgescu D, Salaffi F, Grassi W. Hyaline cartilage involvement in patients with gout and calcium pyrophosphate deposition disease. An ultrasound study. Osteoarthritis Cartilage 2009;17(2):178-181

9 Hellgren L. Association between rheumatoid arthritis and psoriasis in total populations. Acta Rheumatol Scand 1969;15(4):316-326

10 Baker H. Prevalence of psoriasis in polyarthritic patients and their relatives. Ann Rheum Dis 1966;25(3):229-234
11 McGonagle D, Gibbon W, Emery P. Classification of inflammatory arthritis by enthesitis. Lancet 1998;352(9134):1137-1140

12 Healy PJ, Groves C, Chandramohan M, Helliwell PS. MRI changes in psoriatic dactylitis-extent of pathology, relationship to tenderness and correlation with clinical indices. Rheumatology (Oxford) 2008;47(1):92-95

13 Freeston JE, Coates LC, Helliwell PS, et al. Is there subclinical enthesitis in early psoriatic arthritis? A clinical comparison with power Doppler ultrasound. Arthritis Care Res (Hoboken) 2012;64 (10):1617-1621

14 Frediani B, Falsetti P, Storri L, et al. Ultrasound and clinical evaluation of quadricipital tendon enthesitis in patients with psoriatic arthritis and rheumatoid arthritis. Clin Rheumatol 2002;21(3):203-206 